Article original

\title{
Diversité floristique des zones côtières pâturées de la Côte d'Ivoire : cas du cordon littoral Port-Bouët-Grand-Bassam (Abidjan)
}

\author{
Akossoua F. Kouassi ${ }^{*}$, Yao C. Y. Adou ${ }^{1,2}$, Ipou Joseph Ipou ${ }^{1,2}$ \& Kagoyiré KamanzI ${ }^{1}$ \\ 'Université de Cocody-Abidjan, UFR Biosciences, Laboratoire de Botanique, 22 B.P. 582 Abidjan 22, Côte d'Ivoire. \\ ${ }^{2}$ Centre National de Floristique de l'Université de Cocody-Abidjan \\ *Auteur pour les correspondances (E-mail : akossouafifi@yahoo.fr) \\ Reçu le 18-02-2010, accepté le 06-05-2010
}

\begin{abstract}
Résumé
Une étude floristique a été menée sur l'axe Port-Bouët - Grand-Bassam, (Abidjan, Côte d'Ivoire). Elle avait pour objectif de montrer l'intérêt pastoral de la zone. Les relevés de végétation ont été réalisés suivant la méthode stigmatiste classique de Braun-Blanquet et la méthode de tours, afin d'inventorier les espèces floristiques présentes. L'analyse des relevés a permis de dénombrer 190 espèces réparties en 152 genres appartenant à 63 familles botaniques. Parmi ces espèces, 63 plantes fourragères, soit $33,15 \%$ de la flore totale ont été recensées. L'indice de diversité de Shannon pour l'ensemble de la zone d'étude est 4,43. Certaines espèces fourragères, telles que Andropogon canaliculatus, Eriosema glomeratum, Stylosanthes fruticosa, qui sont très appétées par les bovins, sont considérées comme des espèces productrices. L'ensemble de ces espèces à potentialité fourragère, confère à la zone d'étude un intérêt pastoral.
\end{abstract}

Mots clés : flore, fourrage, zone pastorale, zone côtière, Côte d'Ivoire.

\begin{abstract}
Floristic diversity of the grazed coastal zones of Côte d'lvoire: case of the offshore bar Port-BouëtGrand-Bassam (Abidjan).

A floristic study was undertaken on the axis Port-Bouët-Grand-Bassam, in the district of Abidjan (Côte d'Ivoire). It aimed to show the interest of the zone for the pastoralism. The statements of vegetation were carried out using the sigmatist and the turns methods in order to inventory the present floristic species. The analysis of the statements shows 190 species belonging to 152 genera and 63 botanical families. Among them, 63 fodder species i.e. 33,15 $\%$ of the total flora were listed. The index of diversity of Shannon for the whole zone of study is 4.43. The presence of some fodder species such as Andropogon canaliculatus, Eriosema glomeratum, Stylosantes fruticosa very consumed by the bovines are regarded as producing species. These species with fodder potentiality confer to that zone a pastoral interest.
\end{abstract}

Key words: flora, fodder, pastoral zone, coastal zone, Côte d'Ivoire 


\section{Introduction}

En agriculture urbaine, l'élevage, en général, constitue le secteur le mieux représenté après le maraîchage (Mougeot, 2000). L'élevage des ruminants, en particulier, se maintient ou se développe dans les centres urbains ou à proximité de ceux-ci. Cette activité se satisfait du peu d'espace disponible, et est en compétition avec les autres activités agricoles urbaines (maraîchage, horticulture), pour le sol, le travail et le potentiel en biomasse.

En Afrique, dans les pays tels que le Kenya, le Sénégal, le Burkina-Faso, le Niger et bien d'autres, des millions de personnes vivent de l'élevage dans les villes et leurs périphéries (Lee-Smith et al., 1987, Ali et al., 2003). En Côte d'lvoire, bien que l'élevage soit peu développé (Balami, 2003), on constate la présence de petits ou gros élevages à but lucratif ou non. Abidjan, la capitale économique du pays, présente elle aussi des activités d'élevage relativement importantes dans la ville et ses environs. La race bovine élevée est «la race lagunaire ", de petit format et trypanotolérant (C.I.P.E.A., 1979; Landais, 1983; Yapi-Gnaoré et al., 1996).

Les éleveurs de bétail sont confrontés à de nombreux problèmes dont l'alimentation des animaux est le principal. Elle repose, presque exclusivement, sur les ressources naturelles pâturées. Elle est assurée par la biomasse fourragère de la végétation herbacée ou arbustive présente sous forme de poches dans la ville et ses environs. Sur le tronçon Port-Bouët - GrandBassam, site de cette étude, se pratique l'élevage de bovins, également confronté au problème de l'alimentation. Après prospection, cette zone a été choisie à cause de sa proximité avec l'abattoir de Port-Bouët et de sa grande surface de pâturage, relativement aux autres zones de la ville d'Abidjan abritant des élevages semblables de bovins. $\mathrm{Ce}$ type d'élevage est caractérisé par le fait qu'il n'est associé à aucune culture fourragère. De plus, il doit faire face à une réduction progressive d'espace, liée à une forte urbanisation. Il en résulte une occupation progressive des espaces anciennement pâturés et la destruction des végétations herbacées et arbustives.

Le problème de l'alimentation du bétail constitue donc un frein au développement de l'élevage dans ces zones périurbaines. Si rien n'est fait, cette activité sera menacée de disparition alors qu'elle constitue une importante source de revenus pour ces éleveurs, et surtout, qu'elle joue un rôle non négligeable, dans l'approvisionnement en viande des abattoirs de la ville d'Abidjan. En effet, cette source de protéines bovines complète avantageusement celle provenant des bovins importés des pays limitrophes.

Pour faire face à ce problème, une gestion durable des ressources végétales de ces zones s'impose. Pour cela, il est nécessaire de disposer de données de base les concernant. L'analyse floristique de la zone constitue le point de départ de la mise en place de ces données. C'est l'objet, de la présente étude, qui vise à montrer la diversité floristique de la zone pâturée comprise entre Port-Bouët et Grand-Bassam, et l'intérêt de celle-ci pour l'élevage bovin périurbain.

Plus spécifiquement, il s'agira de déterminer la composition et la diversité floristique de la zone, d'identifier les espèces fourragères appétées par les bovins et d'estimer la participation des espèces herbacées fourragères à la production de la biomasse.

\section{Matériel et méthodes}

\subsection{Site d'étude}

L'étude a été menée dans la zone côtière « Est » du cordon littoral, comprise entre les communes de Port- Bouët et de Grand-Bassam (Fig. 1). Cette zone appartient au secteur littoral du domaine guinéen de Guillaumet et Adjanohoun (1971). C'est une savane côtière qui présente une vaste formation herbeuse avec de rares arbustes groupés en buissons ou forts isolés sur un sol sableux. On y rencontre des cocoteraies, des maraîchers, des jachères, un fourré littoral, une exploitation d'anacarde. 


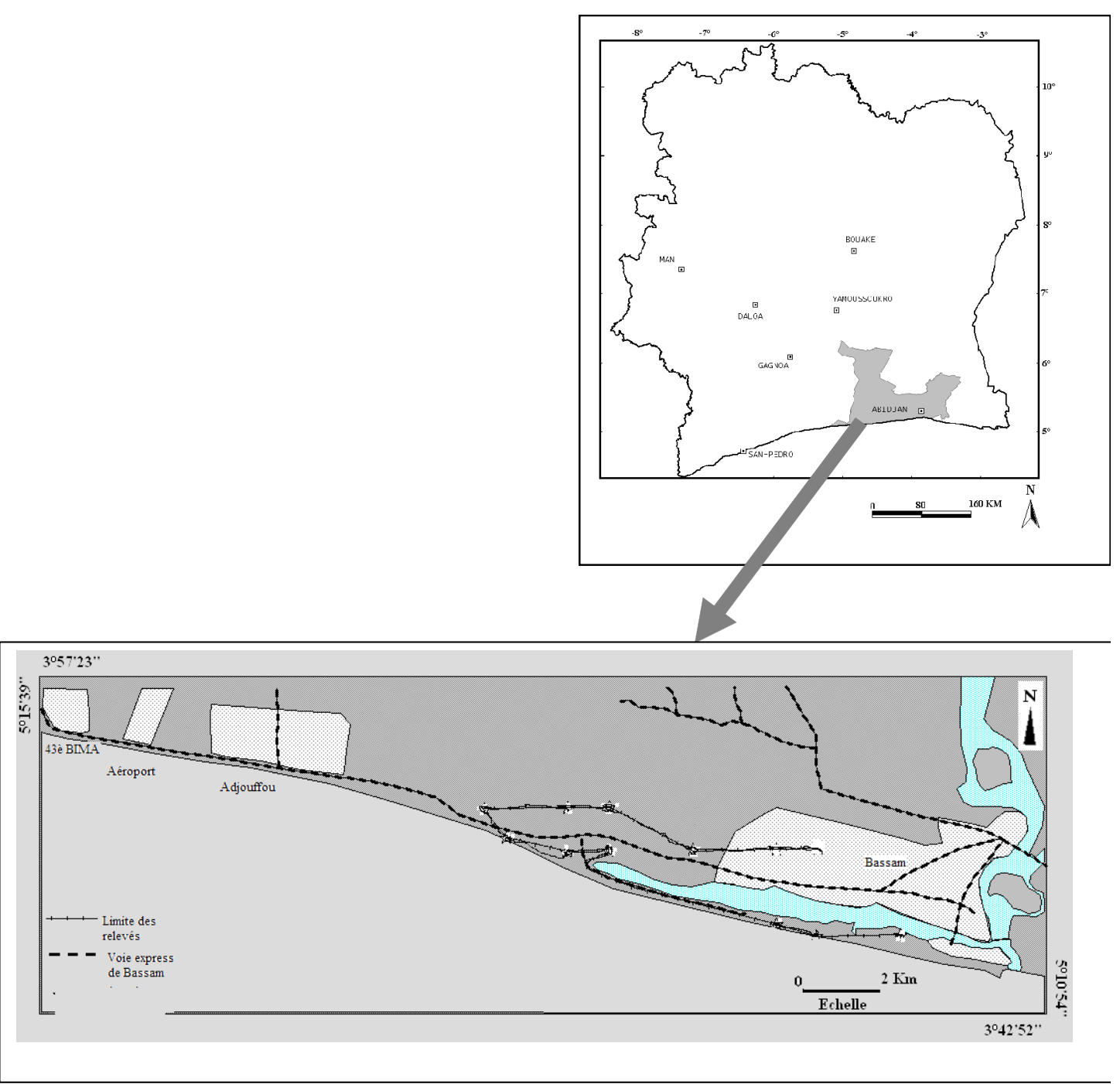

Figure 1: Localisation de la zone d'étude dans la ville d'Abidjan.

\subsection{Matériel biologique}

Le matériel biologique est constitué des échantillons d'espèces végétales récoltées, de l'herbier du Centre National de floristique (C.N.F) de l'Université de Cocody-Abidjan pour des comparaisons en vue d'identifier les échantillons de plantes non déterminées sur le terrain.

\subsection{Matériel technique}

Le matériel technique de cette étude qui a servi à faire l'inventaire floristique de la zone est constitué d'un ruban gradué pour mesurer les aires de relevés, un récepteur GPS (Global Positionning system), pour enregistrer les coordonnées géographiques des différentes zones d'étude, 
une boussole de type SUUNTO pour s'orienter par rapport au parcours des animaux, du papier journal pour la constitution d'herbier, Une bande adhésive pour numéroter les échantillons récoltés et des fiches de collecte des données.

\subsection{Collecte des données}

\subsubsection{Réalisation des relevés}

Le dispositif expérimental est constitué de parcelles choisies sur la base des conditions de drainage du sol, de formations végétales en place et du parcours des animaux lors du pâturage. Ainsi, fourrés littoraux, jachères et cocoteraies ont été échantillonnés selon deux méthodes de relevés.

La première a consisté en un inventaire itinérant suivant le parcours de pâture des bovins. Elle a permis de connaître, les différentes espèces végétales présentes et d'identifier celles qui sont consommées par les bovins (Chicouène, 2000).

La seconde méthode est celle des relevés phytosociologiques réalisés à l'intérieur de placettes de $25 \mathrm{~m} \times 25 \mathrm{~m}$, obtenues à partir de l'aire minimale. Au total, 110 relevés (dont 30 relevés dans les jachères, 30 dans les fourrés littoraux et 50 dans les cocoteraies), ont été effectués à partir de 11 transects tracés dans la direction Sud-Nord. Les relevés n'ont pris en compte que les herbacées et les arbustes présents. Ils ont été réalisés selon la méthode stigmatiste classique de Braun-Blanquet (1932). Cette méthode consiste à dresser la liste des plantes présentes dans un échantillon représentatif et homogène. Pour chaque relevé, sont notées les informations suivantes: localisation, nature du substrat (boue, sable, vase, etc....), date et numéro de récolte des espèces présentes, leurs indices d'abondancedominance. Les relevés ont permis d'établir la liste de toutes les espèces recensées et de calculer la participation de chaque espèce à la production de biomasse. La détermination des espèces est conforme aux différentes flores d'Afrique et de la Côte d'Ivoire (Hutchinson et Dalziel, 1954-1972 ; Aké-Assi, 1984 ; 2001 et 2002). Les espèces non identifiées sur le terrain ont été récoltées et identifiées par comparaison à l'Herbier National de référence du Centre National Floristique ou avec l'aide d'un spécialiste, le Professeur Aké Assi. Cette liste permet de connaître l'enherbement de façon qualitative.

\subsubsection{Enquêtes réalisées auprès des bouviers}

La méthode d'approche est une enquête non structurée réalisée auprès des bouviers, présents sur la zone de pâture des bovins. Le questionnaire comportait des questions ouvertes. L'objectif visé, était de recenser les plantes consommées par les bovins sur l'axe Port-Bouët - Grand-Bassam.

\subsection{Méthode d'analyse des données}

\subsubsection{Diversité qualitative}

L'analyse floristique qualitative (famille, genre, espèces) est réalisée à partir de la liste floristique générale des différents inventaires. Les espèces fourragères énumérées ont été identifiées comme «plantes fourragères » par comparaison à celles énumérées dans la littérature. (Gillet, 1961 ; Ould Soulé, 1995). Elles sont classées dans les différentes catégories de plantes fourragères, selon la classification de HavardDuclos (1967).

\subsubsection{Diversité quantitative}

L'approche quantitative concerne plusieurs points: la fréquence spécifique, le calcul des contributions spécifiques de présence et des indices de diversité.

- la fréquence spécifique de chaque espèce $(F S)$ s'obtient en faisant la somme des présences de chaque espèce dans chaque relevé ; elle est une valeur absolue;

- la contribution spécifique de présence $(C S p)$ correspond à la proportion de chaque espèce présente, exprimée en pourcentage, selon la formule suivante :

$$
C S p i=\frac{F S i}{\sum F S i} \times 100
$$

Plusieurs formules existent et permettent d'évaluer la ressemblance floristique entre des végétations. Parmi celles-ci, le coefficient de 
similitude (Cs) de Sørensen (1948) a été choisi. Il permet de quantifier le degré de ressemblance de deux listes d'espèces de deux sites $A$ et $B$.

$$
c_{s}=\frac{2 a}{(2 a+b+c)}
$$

Dans cette formule, a représente le nombre d'espèces du site $A, b$ le nombre d'espèces du site $B$ et $c$ le nombre d'espèces communes aux deux sites $A$ et $B$.

- Pour caractériser la diversité floristique des milieux de parcours, l'indice de diversité $(H)$ de Shannon (1948) a été utilisé, car c'est l'un des meilleurs estimateurs de la diversité biologique (Magurran, 2005). Cet indice est utilisé comme mesure d'hétérogénéité et prend en compte la régularité d'abondance des espèces (Peet, 1974).

$$
H=-\sum[(C S p(i) / C S) \times(\operatorname{LnCSp}(i) / C S p)]
$$

Dans cette formule, $C S p(i)$ représente la contribution spécifique de présence de l'espèce $i, C S p$ la contribution spécifique de toutes les espèces.

2.5.3. Évaluation de la participation des espèces herbacées fourragères à la production de biomasse.

Selon Daget et Poissonnet (1971), il existe une relation linéaire très étroite entre la composition floristique et la production de biomasse. Ces auteurs ont appelé «espèces productrices» toutes les espèces dont la contribution spécifique de présence $(C S p)$ est au moins égale à $1 \%$. Parmi ces espèces productrices, ils distinguent :

- des espèces très productrices avec $\operatorname{CSp}(i)>4 \pm 1$

- des espèces peu productrices avec $1<\operatorname{CSp}(i)<4 \pm 1$.

Ainsi dans notre étude, cette classification a été utilisée pour connaître les espèces fourragères productrices, qui participent à la production de biomasse.

\section{Résultats}

\subsection{Richesse et diversité floristique de la zone Port-Bouët - Grand-Bassam}

\subsubsection{Diversité qualitative du site d'étude}

Au total, 190 espèces ont été répertoriées. Elles sont réparties en 152 genres appartenant à 63 familles botaniques (Tableau 1). Les angiospermes sont représentées par 60 familles dont les Dicotylédones, avec 49 familles représentant $77,77 \%$ et les Monocotylédones avec 11 familles représentant $17,46 \%$ de la flore totale. Les quatre familles les mieux représentées constituent 38\% de la flore totale. II s'agit de la famille des Poaceae qui, avec 23 espèces, représente à elle seule, $12,11 \%$ de la flore inventoriée; les trois autres familles sont les Fabaceae (19 espèces), les Cyperaceae (17 espèces) et les Rubiaceae (15 espèces), renfermant, respectivement, $10 \%$, $8,95 \%$ et $7,89 \%$ des espèces de la même flore (Annexe 1).

La répartition des genres et des espèces dans les différents types d'écosystème montre que, dans les cocoteraies, huit familles comptent, chacune, plus de cinq espèces. Les familles les plus représentées sont les Poaceae, les Fabaceae, les Cyperaceae et les Rubiaceae. Les espèces Andropogon canaculatus Schumach. (Poaceae), Borreria verticillata (L.) G. Mey. (Rubuaceae) et Cyperus tenax Boeckeler (Cyperaceae) ont été les plus rencontrées.

Dans le fourré littoral et les jachères, cinq familles comptent, en moyenne, cinq espèces chacune. Les familles les mieux représentées sont les Cyperaceae, les Fabaceae et les Poaceae. Les espèces Andropogon canaculatus (Poaceae) et Stylosanthes fruticosa (Retz.) Alston (Fabaceae) dans les jachères et Eriosema glomeratum (Guill. \& Perr.) Hook.f. (Fabaceae) et Brachiaria lata (Schumach.) C.E.Hubbard (Poaceae) dans le Fourré littoral sont les plus rencontrées.

On note la présence de nombreuses plantes ligneuses telles que: Baphia nitida Lodd. (Fabaceae), Chrysobalanus ellipticus Soland. ex Sabine (Chrysobalanaceae), Acacia mangium Willd. (Mimosaceae), Agelaea pentagyna Radlk. (Connaraceae) etc. et la présence de quelques lianes telles que Cucumis sativus $\mathrm{L}$. 
(Cucurbitaceae), Clerodendrum volubile P. Beauv. (Verbenaceae), Albertisia cordifolia (Mangenot \& J. Miège) Forman (Menispermaceae).

Parmi les 190 espèces recensées, 63 sont appétées par les bovins, soit $33,15 \%$ de la flore inventoriée.

\subsubsection{Diversité quantitative de la zone d'étude}

Les indices de diversité ont été calculés pour chaque type de végétation pâturée et pour l'ensemble de la zone d'étude. L'indice de diversité $(H)$ de Shannon pour l'ensemble de la zone d'étude est de 4,43. Au niveau des différents écosystèmes pâturés, cet indice est de 4,29 pour la cocoteraie et est plus élevé que celui du fourré littoral $(3,65)$ et de la jachère $(3,96)$ (Tableau 1$)$.

Les coefficients de similitude de Sørensen calculés entre les différentes zones pâturées sont supérieurs à $50 \%$ (Tableau 2). Ils montrent la ressemblance floristique de ces zones (Fourré littoral, jachères, cocoteraie).

Tableau 1 : Diversité floristique des formations végétales étudiées

\begin{tabular}{lcccc}
\hline & Cocoteraie & Fourré littoral & Jachère & $\begin{array}{c}\text { Ensemble de la zone } \\
\text { d'étude }\end{array}$ \\
\hline Nombre d'espèces & 163 & 71 & 74 & 190 \\
Nombre de familles & 52 & 33 & 27 & 63 \\
Indice de diversité de & 4,29 & 3,65 & 3,96 & 4,43 \\
Shannon $(\mathrm{H})$ & &
\end{tabular}

Tableau 2 : Taux de similitude entre les listes des espèces des formations végétales étudiées (exprimés en \%)

\begin{tabular}{lccc}
\hline & Fourré littoral & Jachères & Cocoteraie \\
\hline Fourré littoral & 100 & 57,14 & 74,04 \\
Jachères & 57,14 & 100 & 73,06 \\
Cocoteraie & 74,04 & 73,06 & 100 \\
\hline
\end{tabular}

\subsection{Caractéristique des espèces fourragères rencontrées}

\subsubsection{Diversité floristique fourragère}

Le classement des différentes espèces fourragères dans les trois catégories fourragères est présenté dans le tableau 3. Les Poaceae fourragères, avec 21 espèces, soit $11,04 \%$ d'espèces fourragères de la flore totale du site, sont les plus nombreuses (Andropogon canaculatus, Eragrostis domingensis (Pers.) Steud.). Elles sont suivies par les autres fourrages (17 espèces), soit $9 \%$ de la flore totale du site (Fimbristylis ferruginea (L.) Vahl, Mariscus cylindristachyus Steud.) et enfin les légumineuses fourragères (12 espèces), qui représentent 6,31\% (Stylosanthes fruticosa, Eriosema glomeratum).

3.2.2. Classification des espèces reconnues par les bouviers comme plantes consommées par les bovins.

Selon les bouviers, la majorité des espèces sont broutées par les bovins. Pour eux, les espèces non consommées sont, pour la plupart, celles qui ont des épines. Ainsi, sur leur indication, nous avons classé les espèces végétales composant le régime alimentaire des bovins, en espèces beaucoup appétées et en espèces peu appétées (Tableau 3). 
Tableau 3 : Liste des espèces fourragères recensées de la zone de pâture Port-Bouët - Grand-Bassam classées par ordre alphabétique

\begin{tabular}{|c|c|c|c|c|c|c|}
\hline \multirow{2}{*}{ Espèces } & \multirow{2}{*}{ Familles } & \multicolumn{3}{|c|}{$\begin{array}{c}\text { Classification en fonction du type de } \\
\text { fourrage }\end{array}$} & \multicolumn{2}{|c|}{$\begin{array}{c}\text { Classification selon les } \\
\text { bouviers }\end{array}$} \\
\hline & & $\begin{array}{l}\text { Légumineuse } \\
\text { fourragère }\end{array}$ & $\begin{array}{l}\text { Poaceae } \\
\text { fourragère }\end{array}$ & $\begin{array}{l}\text { Autres } \\
\text { fourrages }\end{array}$ & $\begin{array}{l}\text { Beaucoup } \\
\text { appétées }\end{array}$ & $\begin{array}{l}\text { Peu } \\
\text { appétées }\end{array}$ \\
\hline Acacia auriculiformis & Mimosaceae & & & & & $x$ \\
\hline Acacia mangium & Mimosaceae & & & & & $x$ \\
\hline Ageratum conyzoides & Asteraceae & & & & & $\mathrm{x}$ \\
\hline Alchornea cordifolia & Euphorbiaceae & & & $\mathrm{x}$ & $\mathrm{x}$ & \\
\hline Andropogon canaliculatus & Poaceae & & $x$ & & $\mathrm{x}$ & \\
\hline Axonopus compressus & Poaceae & & $x$ & & $x$ & \\
\hline Boerhavia diffusa & Nyctaginaceae & & & $x$ & & $x$ \\
\hline Brachiaria lata & Poaceae & & $x$ & & $\mathrm{x}$ & \\
\hline Brachiaria subquadripara & Poaceae & & $\mathrm{x}$ & & $\mathrm{x}$ & \\
\hline Cassia mimosoides & Fabaceae & $x$ & & & & $x$ \\
\hline Cenchrus biflorus & Poaceae & & $x$ & & $x$ & \\
\hline Centrosema pubescens & Fabaceae & $x$ & & & $x$ & \\
\hline Chloris pilosa & Poaceae & & $x$ & & $x$ & \\
\hline Chrysopogon aciculatus & Poaceae & & $x$ & & $x$ & \\
\hline Cleome ciliata & Capparidaceae & & & & & $x$ \\
\hline Crotalaria retusa & Fabaceae & & & & & $x$ \\
\hline Croton lobatus & Euphorbiaceae & & & & & $x$ \\
\hline Cucumis sativus & Cucurbitaceae & & & & & $x$ \\
\hline Cyclosorus striatus & Thelypteridaceae & & & & & $x$ \\
\hline Cynodon dactylon & Poaceae & & $x$ & & $x$ & \\
\hline Cyperus dilatatus & Cyperaceae & & & $x$ & $x$ & \\
\hline Cyperus distans & Cyperaceae & & & $\mathrm{x}$ & $\mathrm{x}$ & \\
\hline Cyperus flabelliformis & Cyperaceae & & & $\mathrm{x}$ & $\mathrm{x}$ & \\
\hline Cyperus rotundus & Cyperaceae & & & $\mathrm{x}$ & $\mathrm{x}$ & \\
\hline Cyperus sphacelatus & Cyperaceae & & & $\mathrm{x}$ & $x$ & \\
\hline Cyperus tenax & Cyperaceae & & & $\mathrm{x}$ & & $\mathrm{x}$ \\
\hline Dactyloctenium aegyptium & Poaceae & & $x$ & & $\mathrm{x}$ & \\
\hline Dalbergia ecastaphyllum & Fabaceae & $x$ & & & & $x$ \\
\hline Daniellia thurifera & Ceasalpiniaceae & & & & & $x$ \\
\hline Desmodium adscendens & Fabaceae & $\mathrm{x}$ & & & & $x$ \\
\hline Desmodium ramosissimum & Fabaceae & $x$ & & & & $x$ \\
\hline Desmodium velutinum & Fabaceae & $x$ & & & $x$ & \\
\hline
\end{tabular}


Tableau 3 (Suite) : Liste des espèces fourragères recensées de la zone de pâture Port-Bouët - Grand-Bassam classées par ordre alphabétique

\begin{tabular}{|c|c|c|c|c|c|c|}
\hline \multirow{2}{*}{ Espèces } & \multirow{2}{*}{ Familles } & \multicolumn{3}{|c|}{$\begin{array}{l}\text { Classification en fonction du type de } \\
\text { fourrage }\end{array}$} & \multicolumn{2}{|c|}{$\begin{array}{c}\text { Classification selon les } \\
\text { bouviers }\end{array}$} \\
\hline & & $\begin{array}{l}\text { Légumineuse } \\
\text { fourragère }\end{array}$ & $\begin{array}{l}\text { Poaceae } \\
\text { fourragère }\end{array}$ & $\begin{array}{l}\text { Autres } \\
\text { fourrages }\end{array}$ & $\begin{array}{l}\text { Beaucoup } \\
\text { appétées }\end{array}$ & $\begin{array}{l}\text { Peu } \\
\text { appétées }\end{array}$ \\
\hline Digitaria horizontalis & Poaceae & & $x$ & & $x$ & \\
\hline Eleusine indica & Poaceae & & $x$ & & $x$ & \\
\hline Eragrostis domingensis & Poaceae & & $x$ & & $x$ & \\
\hline Eriosema glomeratum & Fabaceae & $x$ & & & $x$ & \\
\hline Fimbristylis ferruginea & Cyperaceae & & & $x$ & & $x$ \\
\hline Flagellaria guineensis & Flagellariceae & & & & & $x$ \\
\hline Indigofera hirsuta & Fabaceae & $x$ & & & & $x$ \\
\hline Machaerium Iunatum & Fabaceae & $x$ & & & & $x$ \\
\hline Mariscus alternifolius & Cyperaceae & & & $x$ & $x$ & \\
\hline Mariscus cylindristachyus & Cyperaceae & & & $x$ & $x$ & \\
\hline Mariscus dubius & Cyperaceae & & & $x$ & $x$ & \\
\hline Mariscus flabelliformis & Cyperaceae & & & $x$ & $x$ & \\
\hline Mariscus ligularis & Cyperaceae & & & $x$ & $x$ & \\
\hline Mariscus longibracteatus & Cyperaceae & & & $x$ & $x$ & \\
\hline Merremia tridentata & Conlvolvulaceae & & & $x$ & $x$ & \\
\hline Oldenlandia affinis & Rubiaceae & & & & & $x$ \\
\hline Panicum laxum & Poaceae & & $x$ & & $x$ & \\
\hline Panicum maximum & Poaceae & & $x$ & & $x$ & \\
\hline Panicum repens & Poaceae & & $x$ & & $x$ & \\
\hline Paspalum conjugatum & Poaceae & & $x$ & & $x$ & \\
\hline Passiflora foetida & Passifloraceae & & & $x$ & $x$ & \\
\hline Pennisetum pedicellatum & Poaceae & & $x$ & & $x$ & \\
\hline Pennisetum violaceum & Poaceae & & $x$ & & & $x$ \\
\hline Perotis indica & Poaceae & & $x$ & & $x$ & \\
\hline Pueraria phaseoloides & Fabaceae & $x$ & & & & $x$ \\
\hline Saccharum officinarum & Fabaceae & & $x$ & & & $x$ \\
\hline Sida cordifolia & Malvaceae & & & & $x$ & \\
\hline Sporobolus pyramidalis & Poaceae & & $x$ & & $x$ & \\
\hline Stylosanthes fruticosa & Fabaceae & $x$ & & & $x$ & \\
\hline Triumfetta rhomboidea & Tiliaceae & & & & & $x$ \\
\hline Zornia latifolia & Fabaceae & $x$ & & & $x$ & \\
\hline
\end{tabular}




\subsection{Taux de participation des espèces fourragères à la production de biomasse}

La participation de certaines espèces fourragères à la production de la biomasse est consignée dans le tableau 4. Au total, 13 espèces fourragères ont été identifiées comme " espèces productrices 》.
Ce qui correspond à un taux de participation de $20,63 \%$ à la production de biomasse. Parmi ces espèces, seule une est très productrice ; il s'agit de Andropogon canaculatus, avec une CSp de 4,56 $\%$. Les autres espèces sont peu productrices ; elles ont des contributions spécifiques variant entre 1,09 et $3,62 \%$.

Tableau 4 : Taux de participation des espèces fourragères à la production de biomasse. (CSP : Contribution spécifique due à la présence).

\begin{tabular}{llc}
\hline Classes & Espèces fourragères productrices & CSP (\%) \\
\hline Espèces très productrices & Andropogon canaculatus & 4,56 \\
\hline & Cleome ciliata & 3,62 \\
& Oldenlandia affinis & 3,57 \\
& Ageratum conyzoides & 2,75 \\
& Flagellaria guineensis & 2,49 \\
& Stylosanthes fruticosa & 2,43 \\
& Eriosema glomeratum & 1,66 \\
& Digitaria horizontalis & 1,55 \\
& Sida cordifolia & 1,45 \\
& Chrysopogon aciculatus & 1,29 \\
& Cyperus tenax & 1,29 \\
& Eragrostis domingensis & 1,19 \\
& Triumfetta rhomboidea & 1,09 \\
\hline
\end{tabular}

\section{Discussion}

L'inventaire floristique réalisé par Adjanohoun (1962) dans son étude phytosociologique des savanes de basse Côte d'Ivoire (Savanes lagunaires) comprend 288 espèces recensées. Il a été réalisé dans plusieurs savanes lagunaires de la Côte d'Ivoire, à l'exception de celle qui se trouve sur le cordon littoral, particulièrement dans sa partie comprise entre Port-Bouët et GrandBassam et qui fait l'objet de cette étude. Au total, 190 espèces végétales appartenant à 63 familles botaniques ont été recensées. Ce nombre est inférieur à celui obtenu par Adjanohoun (1962). Cela peut s'expliquer par le fait que notre étude n'a pris en compte que l'espace du cordon littoral pâturé par le bétail. Les zones non accessibles aux bovins n'ont pas été inventoriées, de même, la zone inventoriée ne couvre pas la même superficie que celle de Adjanohoun (1962), qui a regroupé plusieurs savanes lagunaires de la Côte d' Ivoire.

Concernant la composition floristique, on constate que la famille des Poaceae est la plus représentée, suivie de celles des Légumineuses et des Cyperaceae. La forte proportion des Poaceae dans la zone peut s'expliquer par le fait que ces taxons possèdent une très grande possibilité de tallage et une plus grande vitesse de repousse après broutage, lorsque les 
conditions du milieu sont favorables (Salette, 1970). Ces résultats corroborent ceux de Adjanohoun (1962) qui, dans son étude a trouvé que les espèces de la famille des Poaceae étaient les plus nombreuses, suivies de celles des Légumineuses et des Cyperaceae.

Plusieurs espèces fourragères, par comparaison à celles citées par Ould Soulé (1995) ont été dénombrées. Au nombre de celles-ci, les Poaceae fourragères sont représentées surtout par Andropogon canaculatus, Brachiaria lata, Axonopus compressus (Sw). P. Beauv., Cenchrus biflorus Roxb., Chrysopogon aciculatus (Retz.) Trin.

, Eragrostis domingensis, Panicum maximum Jacq., etc. qui sont consommées par les bovins. Elles sont, en général, présentes pendant toute l'année et résistent au broutage et au piétinement des bovins. Les Graminées, en général, ont des potentialités de production et sont très appétées par les bovins (Heldin, 1965). La forte proportion des Poaceae peut aussi s'expliquer par le fait que, en plus d'être des espèces fourragères consommées et prisées par les ruminants, elles sont résistantes aux aléas climatiques et au broutage et sont rarement atteintes de maladies cryptogamiques (Salette, 1970).

Concernant les Légumineuses fourragères, elles sont d'excellents fourrages et sont représentées dans notre étude par Stylosanthes fruticosa, Zornia latifolia Sm., Centrosema pubescens Benth. et Eriosema glomeratum. Stylosanthes fruticosa est la plus représentative avec une contribution spécifique de présence supérieure à $2 \%$. Selon Boudet (1963), la meilleure valeur fourragère des légumineuses fait que les plus productives d'entre elles, en particulier Stylosanthes fruticosa dans cette étude, sont susceptibles d'être retenues dans un système intensif.

En plus de ces deux catégories de plantes fourragères, d'autres espèces végétales appartenant à des familles autres que les Poaceae et les Légumineuses, contribuent aussi à l'alimentation des animaux. Elles ont été nommées « autres fourrages». Elles sont souvent très appétées et jouent un rôle important dans l'alimentation du bétail. A titre d'exemple, Fimbristylis ferruginea, Mariscus flabelliformis H.B. et K. var. flabelliformis, Mariscus cylindristachyus sont consommées par les bovins (Ould Soulé, 1995) et sont citées par les bouviers comme d'excellents fourrages. Toutes ces espèces fourragères citées dans cette étude montrent que les espaces non exploités jouent un rôle très important dans l'alimentation des ruminants en Afrique (Guerin, 1977). Par ailleurs, l'ensemble des espèces recensées permet de connaître la composition floristique de la zone d'étude et d'affirmer que les plantes fourragères rencontrées sont, pour la plupart, très appétées par les bovins.

Les indices de diversité floristique constituent des critères objectifs pour apprécier la diversité d'une communauté végétale (Ramade, 1994). L'indice de diversité de Shannon pour l'ensemble de la zone d'étude est de 4,43. Cet indice est plus élevé pour la végétation de cocoteraie que pour celle du fourré littoral et des jachères. Les cocoteraies abritent un nombre élevé d'espèces végétales et sont les plus pâturés à cause de l'ombrage qu'offrent ces cocotiers aux animaux. L'indice $(H)$ de Shannon qui est plus élevé dans la zone la plus pâturée corrobore celui de Rakotoarimanana et Grouzis (2006) trouvé dans le Sud-Ouest de Madagascar. Ces auteurs rapportent que les zones les plus pâturées entraînent une augmentation de la diversité floristique (Hélevé). En effet, de nombreux travaux au Sahel et dans les savanes soudaniennes concluent à un maintien ou une augmentation de la diversité floristique herbacée, en cas d'exploitation pastorale importante (César, 1992 ; Fournier et al., 2000; Koita \& Bodian, 2000). Toutefois, ces résultats ne s'accordent pas avec ceux de Hiernaux (1998) et de Rakotoarimanana et al. (2008) qui montrent que le pâturage diminue significativement les indices de diversité.

\section{Conclusion}

Au terme de cette étude, l'analyse floristique de la zone Port-Bouët - Grand-Bassam a permis de connaître la composition floristique de la zone pâturée, d'identifier les espèces fourragères appétées par les bovins et d'estimer la participation des espèces herbacées fourragères à la production de la biomasse. Cette analyse révèle une diversité floristique aussi bien qualitative que quantitative. Les familles des Poaceae, Fabaceae, Cyperaceae et Rubiaceae sont les mieux représentées. Les espèces telles que Cleome ciliata Schumach. \& Thonn. (Capparaceae), Panicum maximum (Poaceae), 
Stylosanthes fruticosa (Fabaceae), Eriosema glomeratum (Fabaceae) sont appelées " espèces productrices " avec des CSp comprises entre $1 \%$ et $4 \%$. L'espèce Andropogon canaculatus (Poaceae) présente une CSp supérieur à $4 \%$; elle est donc « très productrice». Toutes ces espèces citées contribuent de manière significative à la production de biomasse. Aussi, avec l'aide des bouviers, nous avons identifié les espèces végétales composant le régime alimentaire des bovins. Celles-ci ont été classées en espèces beaucoup appétées et en espèces peu appétées. Au regard de tout cela, nous pouvons dire que la zone Port-Bouët - Grand-Bassam joue un rôle important dans l'alimentation des bovins et serait propice à abriter l'élevage de grande taille. Cet élevage constituerait une importante source de revenus et jouerait un grand rôle dans l'approvisionnement en viande des abattoirs de la ville d'Abidjan.

\section{Références citées}

Adjanohoun E.J., 1962. Etude phytosociologique des savanes de basse Côte d'Ivoire (Savane lagunaire). Vegetatio., 11 (1-2): 1-38.

Aké-Assi L., 1984. Flore de la Côte d'Ivoire. Etude descriptive et biogéographique avec quelques notes ethnobotaniques. Thèse de Doctorat d'Etat, Université d'Abidjan, Côte d'Ivoire. 6 vol. ; 1206 pp

Aké-Assi L., 2001. Flore de la Côte d'Ivoire: catalogue systématique, biogéographie et écologie. Volume 1: Mémoire de Botanique systématique. Conservatoire et Jardin Botanique de Genève ; Boisseria 57 ; 396 pp.

Aké-Assi L., 2002. Flore de la Côte d'lvoire: catalogue systématique, biogéographie et écologie. Volume 2: Mémoire de Botanique systématique. Conservatoire et Jardin Botanique de Genève ; Boisseria 58 ; 441 pp.

Ali L., Van den Bossche P. \& Thys E., 2003. Enjeux et contraintes de l'élevage urbain et périurbain des petits ruminants à Maradi au Niger : quel avenir? Revue Élev. Méd. vét. Pays trop., 56 (1-2) : 73-82.

Balami D. H., 2003. Le rôle de la filière bétail dans la compétitivité des pays ouest- africains. In : Club du Sahel. L'Afrique de l'Ouest et la Mondialisation : les Facteurs de la Compétitivité.

Boudet G., 1963. Pâturages et plantes fourragères en République de Côte d'Ivoire - I.E.\& M.V.T., 102 pp.

Braun-Blanquet J., 1932. Plant sociology. The study of plant communities (English translation of «pflazensoziologie» by G. D. Fuller and H.S. Conard) Université de chicago (USA), 439 pp.

César J., 1992. Etude de la production biologique des savanes de la Côte d'Ivoire et son utilisation par l'homme. Biomasse, valeur pastorale et production fourragère. Doct. Etat, université Pierre et Marie Curie, Paris, France, 672 pp.

Chicouène D., 2000. Evaluation du peuplement des mauvaises herbes en végétation dans une parcelle: Protocoles rapides pour un usage courant. Phytoma- Défense des cultures, 524 : 18-23.

C.I.P.E.A., 1979. Centre International pour I'Elevage en Afrique. Monographie 2. Le bétail trypanotolérant d'Afrique Occidentale et Centrale. Tome 1, Situation Générale: Addis Abeba, 155 pp.

Daget P. \& Poissonet J., 1971. Une méthode d'analyse phytosociologique des prairies. Critères d'application. Annales Agronomiques, 22 : 5-41.

Fournier A., Yoni M. \& Zombre P., 2000. Les jachères à Andropogon gayanus en savane soudanienne dans l'ouest du Burkina Faso: flore, structure, déterminants et fonction dans l'écosystème. Etude Flore végétation. Burkina Faso, 5: 3-32.

Gillet H., 1961. Pâturages sahéliens. Le ranch de l'Ouadi Rimé. 1 vol. Paris, Journ. Agric. Trop. Bot. Appl., 210 pp.

Guerin H., 1977. Exploitation de pâturages de Stylosanthes et de Brachiaria en culture sèche. Campagne 1975-1976.

Guillaumet J.L. \& Adjanohoun E., 1971. La végétation de la Côte d'Ivoire. In le milieu naturel de la Côte d'Ivoire. ORSTOM

Havard-Duclos B., 1967. Les plantes fourragères tropicales. Collection Techniques Agricoles 
et Productions Agricoles. Paris, Maisonneuve et Larose, $397 \mathrm{pp}$.

Heldin L., 1965. Fourrages tropicaux - Fourrages 22 : 185-197.

Hiernaux P., 1998. Effects of grazing on plant species composition and spatial distribution in rangelands of the Sahel. Plant Ecology, 33: 387-399.

Hutchinson J. \& Daziel J. M., 1954-1972 Flora of West Tropical Africa, ed. 2, revue par Keay R. W. J. et Hepper F. N. Crown Agents for oversea government and administrations London, 3 volumes.

Koita B. \& Bodian A., 2000. Evolution de la diversité végétale avec le temps de jachère en zone soudanienne au Sénégal. In : Floret C., Pontanier R., éds, La jachère en Afrique tropicale. Montrouge, France, John Libbey Eurotext, pp. 408-414.

Landais E. 1983. Analyse des systèmesd'élevage bovin sédentaire du Nord de la Côte d'Ivoire. Thèse Doct. Etat. IEMVT, Maisons-Alfort, France. 2 vol., pp. 759.

Lee-Smith D., Manundu M., Davinder L., \& Gathuru P.K., 1987. 'Urban Food Production and the Cooking Fuel Situation in Urban Kenya', Mazingira Institute, Nairobi, Kenya.

Magurran A. E., 2005. Measuring Biological Diversity. Blackwell Publishing, UK, 256 pp.

Mougeot L. J. A., 2000. Urban agriculture: definition, presence, potential and risks. In: Bakker N. et al., Growing cities, growing food: urban agriculture on the policy agenda, a reader on urban agriculture. Feldafing, Allemagne, Des-Etc. concept and definition. UA-Mag. 1: 5-7.
Ould Soulé A., 1995. Utilisation de la flore de la Mauritanie. Cours de 384 Postgrado Université de Nouakchott/Université de Barcelone, $48 \mathrm{pp}$.

Peet R. K., 1974. The measurement of species diversity. Annual Review of Ecology and Systematics, 5: 285-307.

Rakotoarimanana V. \& Grouzis M., 2006. Influence of fire and of grazing on the richness and the floristic diversity of a savanna dominated by Heteropogon contortus in the southwest of Madagascar (region of Sakaraha), Candollea. 61 (1): 167-188.

Rakotoarimanana V., Gondard H., Ranaivoarivelo N. \& Carriere S., 2008. Influence des pâturages sur la diversité floristique, la production et la qualité fourragères d'une savane des Hautes Terres malgaches (région de Fianarantsoa). Sécheresse 19 (1): 39-46.

Ramade F., 1994. Eléments d'Ecologie. Ecologie fondamentale 2. Ediscienceinternational, Paris, 579 pp.

Salette J.O., 1970. Les cultures fourragères tropicales et leurs possibilités d'intensification. Fourrages 43: 91-105.

Shannon C.E., 1948. A mathematical theory of communication Bell System Technical Journal 27: 379-423.

Sørensen T., 1948. A method of establishing groups of equal amplitude in plant sociology based on similarity of species content. $\mathrm{K}$. Danske Vidensk. Selsk . 5 :1-34.

Yapi-Gnaore, C. V., B. A. Oya \& Ouattara Z., 1996. Revue de la situation des races d'animaux domestiques de Côte d'Ivoire.Animal Genetic Resources Information. 19: 99-118. 
Annexe 1: Liste des espèces recensées de la zone de pâture Port-Bouët - Grand-Bassam classées par ordre alphabétique.

\begin{tabular}{|c|c|c|c|c|}
\hline \multirow[b]{2}{*}{ Espèces } & \multirow[b]{2}{*}{ Familles } & \multicolumn{3}{|c|}{ Formations végétales } \\
\hline & & Cocoteraie & \begin{tabular}{|l} 
Fourré \\
littoral
\end{tabular} & Jachère \\
\hline Abrus precatorius $\mathrm{L}$. & Fabaceae & $\mathrm{x}$ & $x$ & $\mathrm{x}$ \\
\hline Acacia auriculiformis A. Cunningham ex Benth. & Mimosaceae & $x$ & & \\
\hline Acacia mangium Willd. & Mimosaceae & $x$ & $x$ & $x$ \\
\hline Agelaea pentagyna Radlk. & Connaraceae & $x$ & $x$ & \\
\hline Ageratum conyzoides L. & Asteraceae & $x$ & $x$ & $x$ \\
\hline Albertisia cordifolia (Mangenot \& J.Miège) Forman & Menispermaceae & $\mathrm{x}$ & $\mathrm{x}$ & \\
\hline Albertisia scandens (Mangenot \& Miege) Forman & Menispermaceae & $x$ & & \\
\hline Alchornea cordifolia (S.\& Th) Mûll.Arg.Macbr & Euphorbiaceae & $x$ & & $x$ \\
\hline Amaranthus viridis $\mathrm{L}$. & Amaranthaceae & & $x$ & $x$ \\
\hline Ananas comosus (L.) Merr. & Bromeliaceae & $x$ & & $x$ \\
\hline Ancistrocladus barteri Scott-Elliot & Ancistrocladaceae & $x$ & & \\
\hline Andropogon canaliculatus Schumach. & Poaceae & $x$ & $x$ & $x$ \\
\hline Anthocleista nobilis G. Don. & Loganiaceae & $x$ & $x$ & \\
\hline Asystasia gangetica (L.) T. Anders. & Acanthaceae & $x$ & & \\
\hline Axonopus compressus Sw. P. Beauv. & Poaceae & $x$ & & \\
\hline Azadirachta indica A. Juss & Meliaceae & $x$ & & \\
\hline Baphia bancoensis Aubrev. & Fabaceae & $x$ & & \\
\hline Baphia nitida Lodd & Fabaceae & $x$ & $x$ & \\
\hline Boerhavia diffusa L. & Nyctaginaceae & $x$ & & \\
\hline Borreria verticillata (L.) G.Mey. & Rubiaceae & $x$ & $x$ & $x$ \\
\hline Brachiaria lata (Schumach.) C.E.Hubbard & Poaceae & $x$ & $x$ & \\
\hline Brachiaria subquadripara (Trin.) Hitchc. & Poaceae & $x$ & & $x$ \\
\hline Byrsocarpus coccineus Schumach. & Connaraceae & $x$ & $x$ & \\
\hline Capparis erythrocarpos I.sert & Capparidaceae & & $x$ & \\
\hline Carpolobia lutea G. Don & Polygalaceae & $x$ & & $x$ \\
\hline Cassia mimosoides $\mathrm{L}$. & Caesalpiniaceae & $x$ & $x$ & $x$ \\
\hline Cassia occidentalis L. & Caesalpiniaceae & $x$ & & \\
\hline Cassipourea barteri N.E. Br. & Rhizophoraceae & $x$ & $x$ & $x$ \\
\hline Cassytha filiformis $\mathrm{L}$. & Lauraceae & $x$ & & $x$ \\
\hline Catharanthus roseus G. Don & Apocynaceae & $x$ & & $x$ \\
\hline Cenchrus biflorus Roxb. & Poaceae & & $x$ & $x$ \\
\hline
\end{tabular}


Annexe 1 (Suite): Liste des espèces recensées de la zone de pâture Port-Bouët - Grand-Bassam classées par ordre alphabétique.

\begin{tabular}{|c|c|c|c|c|}
\hline \multirow[b]{2}{*}{ Espèces } & \multirow[b]{2}{*}{ Familles } & \multicolumn{3}{|c|}{ Formations végétales } \\
\hline & & Cocoteraie & $\begin{array}{l}\text { Fourré } \\
\text { littoral }\end{array}$ & Jachère \\
\hline Centrosema pubescens Benth. & Fabaceae & $\mathrm{x}$ & $\mathrm{x}$ & $\mathrm{x}$ \\
\hline Chloris pilosa Schumach. & Poaceae & & & $x$ \\
\hline Chromolaena odorata (L.) King \& H.E. Robins. & Asteraceae & $\mathrm{x}$ & $x$ & $x$ \\
\hline Chrysobalanus icaco L. subsp. Icaco L. & Chrysobalanaceae & $\mathrm{x}$ & & $\mathrm{x}$ \\
\hline Chrysopogon aciculatus (Retz.) Trin. & Poaceae & $x$ & & $x$ \\
\hline Citrullus vulgaris Eckl. \& Zeyh & Cucurbitaceae & $x$ & $x$ & \\
\hline Clappertonia ficifolia (Willd.) Decne. & Tiliaceae & $x$ & $\mathrm{x}$ & \\
\hline Cleome ciliata Schumach \& Thonn. & Capparidaceae & $x$ & $x$ & \\
\hline Cleome viscosa $\mathrm{L}$. & Capparidaceae & $\mathrm{x}$ & $\mathrm{x}$ & \\
\hline Clerodendrum volubile P.Beauv. & Verbenaceae & $x$ & & \\
\hline Cnestis ferruginea DC. & Connaraceae & $x$ & & \\
\hline Commelina diffusa Burm. f. & Commelinaceae & $x$ & $x$ & \\
\hline Commelina erecta L. & Commelinaceae & $x$ & & \\
\hline Crotalaria pallida Aiton var. Pallida Aiton & Fabaceae & $\mathrm{x}$ & & \\
\hline Crotalaria retusa L. & Fabaceae & $x$ & $x$ & $x$ \\
\hline Croton lobatus L. & Euphorbiaceae & & & $x$ \\
\hline Cucumis sativus $\mathrm{L}$. & Cucurbitaceae & $x$ & & \\
\hline Cyclosorus striatus (Schum.) Ching & Thelypteridaceae & $x$ & $x$ & \\
\hline Cynodon dactylon (L.) Pers & Poaceae & $\mathrm{x}$ & & \\
\hline Cyperus dilatatus Schumach. \& Thonn. & Cyperaceae & & $x$ & \\
\hline Cyperus distans L. f. & Cyperaceae & $x$ & $x$ & \\
\hline Cyperus rotundus $\mathrm{L}$. & Cyperaceae & $x$ & $x$ & $x$ \\
\hline Cyperus sphacelatus Rottb. & Cyperaceae & $x$ & $x$ & \\
\hline Cyperus tenax Boeckeler & Cyperaceae & $\mathrm{x}$ & & $\mathrm{x}$ \\
\hline Cyrtosperma senegalense (Schott) Engl. & Araceae & $x$ & $\mathrm{x}$ & \\
\hline Dactyloctenium aegyptium (L.) Willd. & Poaceae & & & $x$ \\
\hline Dalbergia ecastaphyllum (L.) Taubert & Fabaceae & $x$ & $x$ & $x$ \\
\hline Daniellia thurifera Bennett & Caesalpiniaceae & $\mathrm{x}$ & & \\
\hline Desmodium ascendens (Sw.) DC. & Fabaceae & $x$ & & \\
\hline
\end{tabular}


Annexe 1 (Suite): Liste des espèces recensées de la zone de pâture Port-Bouët - Grand-Bassam classées par ordre alphabétique.

\begin{tabular}{|c|c|c|c|c|}
\hline \multirow[b]{2}{*}{ ESPECES } & \multirow[b]{2}{*}{ Familles } & \multicolumn{3}{|c|}{ Formations végétales } \\
\hline & & Cocoteraie & \begin{tabular}{|l} 
Fourré \\
littoral
\end{tabular} & Jachère \\
\hline Desmodium ramosissimum G.Don & Fabaceae & $x$ & & $x$ \\
\hline Desmodium velutinum (Willd.) DC. & Fabaceae & $x$ & & \\
\hline Digitaria horizontalis Willd. & Poaceae & $x$ & & $x$ \\
\hline Diodia rubricosa Hiern & Rubiaceae & $x$ & & $x$ \\
\hline Diodia sarmentosa Sw. & Rubiaceae & $x$ & $x$ & \\
\hline Diospyros tricolor (Schumach. \& Thonn.) Hiern & Ebenaceae & & $x$ & \\
\hline Elaeis guineensis Jacq. & Arecaceae & $x$ & & \\
\hline Eleusine indica (L.) Gaertn. & Poaceae & $x$ & $x$ & \\
\hline Emilia praetermissa Milne-Redh & Asteraceae & $x$ & & $x$ \\
\hline Emilia sonchifolia (L.) DC. & Asteraceae & $x$ & & $x$ \\
\hline Eragrostis domingensis (Pers.) Steud. & Poaceae & $x$ & $x$ & $x$ \\
\hline Eriosema glomeratum (Guill. \& Perr.) Hook.f. & Fabaceae & $\mathrm{x}$ & $x$ & \\
\hline Eucalyptus camaldulensis Dehnhardt & Myrtaceae & $x$ & & \\
\hline Euphorbia hirta L. & Euphorbiaceae & $x$ & & \\
\hline Euphorbia hyssopifolia L. & Euphorbiaceae & & & $x$ \\
\hline Ficus exasperata Vahl. & Moraceae & $x$ & & \\
\hline Ficus ovata Vahl. & Moraceae & $x$ & & $x$ \\
\hline Ficus trichopoda Baker & Moraceae & $x$ & & \\
\hline Fimbristylis ferruginea (L.) Vahl. & Cyperaceae & $\mathrm{x}$ & & $x$ \\
\hline Flagellaria guineensis Schumach. & Flagellariaceae & $x$ & $x$ & $x$ \\
\hline Fuirena umbellata Rottb. & Cyperaceae & $x$ & & \\
\hline Gaertnera paniculata Benth. & Rubiaceae & $x$ & & \\
\hline Glinus oppositifolius (L.) Aug.DC. & Aizoaceae & $x$ & & \\
\hline Heliconia psittacorum L. f. & Heliconiaceae & $\mathrm{x}$ & & $\mathrm{x}$ \\
\hline Heterotis rotundifolia (Sm.) Jacq.-Fél. & Melastomataceae & & & $x$ \\
\hline Hibiscus asper Hook.f. & Malvaceae & $x$ & & $x$ \\
\hline Hibiscus sabdariffa L. & Malvaceae & $x$ & & $x$ \\
\hline Imperata cylindrica (L.) Beauv. & Poaceae & $x$ & & \\
\hline Indigofera hirsuta L. & Fabaceae & $\mathrm{x}$ & & $x$ \\
\hline Ipomoea asarifolia (Desr.) Roem. \& Schult. & Convolvulaceae & $x$ & & \\
\hline Ipomoea cairica (L.) Sweet & Convolvulaceae & $x$ & & \\
\hline
\end{tabular}


Annexe 1 (Suite): Liste des espèces recensées de la zone de pâture Port-Bouët - Grand-Bassam classées par ordre alphabétique.

\begin{tabular}{|c|c|c|c|c|}
\hline \multirow[b]{2}{*}{ Espèces } & \multirow[b]{2}{*}{ Familles } & \multicolumn{3}{|c|}{ Formations végétales } \\
\hline & & Cocoteraie & $\begin{array}{l}\text { Fourré } \\
\text { littoral }\end{array}$ & Jachère \\
\hline Ipomoea involucrata P. Beauv. & Convolvulaceae & $\mathrm{x}$ & & \\
\hline Ipomoea mauritiana Jacq. & Convolvulaceae & $x$ & & $x$ \\
\hline Ipomoea stolonifera (Cirillo) J.F. Gmel. & Convolvulaceae & & $x$ & \\
\hline Isonema smeathmannii Roem. \& Schult. & Apocynaceae & $x$ & & \\
\hline Jatropha curcas L. & Euphorbiaceae & $x$ & $x$ & \\
\hline Jussiaea linifolia Vahl. & Onagraceae & & & $x$ \\
\hline Kohautia confusa (Hutch. \& Dalziel) Bremek. & Rubiaceae & $x$ & $x$ & \\
\hline Kyllinga peruviana Lam. & Cyperaceae & $x$ & & $x$ \\
\hline Lannea welwitschii (Hiern) Engl & Anacardiaceae & $x$ & & \\
\hline Lantana camara L. & Verbenaceae & $x$ & & $x$ \\
\hline Leptoderris miegei Ake Assi \& Mangenot & Fabaceae & $x$ & $x$ & $x$ \\
\hline Ludwigia octovalvis (Jacq.) Raven & Onagraceae & $x$ & $x$ & $x$ \\
\hline Machaerium lunatum (L. f.) Ducke & Fabaceae & $x$ & & \\
\hline Mangifera indica $\mathrm{L}$. & Anacardiaceae & $x$ & & \\
\hline Manotes longiflora Baker & Connaraceae & $x$ & & \\
\hline Mariscus cylindristachyus Steud. & Cyperaceae & $x$ & & \\
\hline Mariscus dubius (Rottb.) Kük. ex C.E.C.Fisch. & Cyperaceae & $x$ & & \\
\hline Mariscus alternifolius Vahl. & Cyperaceae & & & $x$ \\
\hline Mariscus flabelliformis H.B.K var. flabelliformis & Cyperaceae & $x$ & & \\
\hline Mariscus ligularis (L.) Urban & Cyperaceae & $x$ & $x$ & \\
\hline Mariscus longibracteatus Cherm. & Cyperaceae & $x$ & $x$ & \\
\hline Maytenus serrata (Hochst.) R.Wilczek & Celastraceae & & $x$ & \\
\hline Melastomastrum cornifolium (Benth.) Jacq.-Fél. & Melastomataceae & $x$ & & \\
\hline Melinis repens (Willd.) Zirka subsp. Repens(Willd.) Zirka & Poaceae & & & $x$ \\
\hline Melochia corchorifolia L. & Sterculiaceae & $x$ & $x$ & \\
\hline Merremia tridentata (L.) Hallier f. & Convolvulaceae & $x$ & & \\
\hline Mesanthemum radicans (Benth.) Körn. & Eriocaulaceae & & & $x$ \\
\hline Microgramma owariensis (Desv.) Alston & Polypodiaceae & $x$ & & \\
\hline Milicia excelsa (Welw.) C.C.Berg & Moraceae & $x$ & & \\
\hline Mimosa pudica L. & Mimosaceae & $x$ & & \\
\hline Mollugo nudicaulis Lam. & Aizoaceae & $x$ & & \\
\hline Morinda longiflora G.Don & Rubiaceae & $x$ & $x$ & \\
\hline Morinda lucida Benth. & Rubiaceae & $\mathrm{x}$ & & \\
\hline
\end{tabular}


Annexe 1 (Suite): Liste des espèces recensées de la zone de pâture Port-Bouët - Grand-Bassam classées par ordre alphabétique.

\begin{tabular}{|c|c|c|c|c|}
\hline \multirow[b]{2}{*}{ ESPECES } & \multirow[b]{2}{*}{ Familles } & \multicolumn{3}{|c|}{ Formations végétales } \\
\hline & & Cocoteraie & $\begin{array}{l}\text { Fourré } \\
\text { littoral }\end{array}$ & Jachère \\
\hline Napoleonaea vogelii Hook. \& Planch. & Napoleonaeaceae & $\mathrm{x}$ & $x$ & \\
\hline Nephrolepis biserrata (Sw.) Schott & Davalliaceae & $x$ & & \\
\hline Newbouldia laevis (P.Beauv.) Seem. ex Bureau & Bignoniaceae & $x$ & $x$ & \\
\hline Ochtocosmus africanus Hook. $\mathrm{f}$. & Ixonanthaceae & $x$ & & \\
\hline Oldenlandia affinis (Roem. \& Schult.) DC. & Rubiaceae & $x$ & & $x$ \\
\hline Oldenlandia corymbosa L. & Rubiaceae & $x$ & & \\
\hline Oldenlandia herbacea (L.) Roxb. & Rubiaceae & $x$ & $x$ & \\
\hline Opuntia ficus-indica (L.) P. Mill. & Cactaceae & & $x$ & \\
\hline Otomeria guineensis Benth. & Rubiaceae & $x$ & & \\
\hline Panicum laxum Sw. & Poaceae & $x$ & $x$ & $x$ \\
\hline Panicum maximum Jacq. & Poaceae & $x$ & & $x$ \\
\hline Panicum repens $\mathrm{L}$. & Poaceae & $x$ & $x$ & $x$ \\
\hline Parkia biglobosa (Jacq.) R.Br. ex G.Don & Mimosaceae & $x$ & & \\
\hline Paspalum conjugatum Berg. & Poaceae & $x$ & & \\
\hline Passiflora foetida L. & Passifloraceae & $x$ & $x$ & \\
\hline Pedalium murex $\mathrm{L}$. & Pedaliaceae & & & $x$ \\
\hline Pennisetum pedicellatum Trin. & Poaceae & $x$ & & $x$ \\
\hline Pennisetum violaceum (Lam.) Rich. & Poaceae & $x$ & & \\
\hline Pentodon pentandrus (K. Schum.) Vatke & Rubiaceae & $x$ & & \\
\hline Perotis indica (L.) Kuntze & Poaceae & $x$ & & \\
\hline Phoenix reclinata Jacq. & Arecaceae & & $x$ & \\
\hline Phyllanthus amarus Schumacher & Euphorbiaceae & $x$ & $x$ & $x$ \\
\hline Phyllanthus pentandrus Schumach. \& Thonn. & Euphorbiaceae & $x$ & & $x$ \\
\hline Phymatodes scolopendria (Burm. f.) Ching & Polypodiaceae & $x$ & $x$ & \\
\hline Pristimera paniculata (Vahl.) N.Hallé & Hippocrateaceae & $x$ & & \\
\hline Psidium guajava L. & Myrtaceae & $x$ & $x$ & $x$ \\
\hline Pteridium aquilinum (L.) Kuhn & Dennstaedtiaceae & $x$ & & \\
\hline Pueraria phaseoloides (Roxb.) Benth. & Fabaceae & & $x$ & \\
\hline Rauvolfia vomitoria Afzel. & Apocynaceae & $x$ & $x$ & $x$ \\
\hline Rhynchospora holoschoenoides (Rich.) Herter & Cyperaceae & $x$ & $x$ & $x$ \\
\hline Rinorea ilicifolia (Welw. ex Oliv.) Kuntze & Violaceae & $x$ & & \\
\hline Ritchiea capparoides (Andr.) Britt. & Capparidaceae & $x$ & & \\
\hline Saba senegalensis (A. DC.) Pichon & Apocynaceae & $x$ & & \\
\hline
\end{tabular}


Annexe 1 (Suite): Liste des espèces recensées de la zone de pâture Port-Bouët - Grand-Bassam classées par ordre alphabétique.

\begin{tabular}{|c|c|c|c|c|}
\hline \multirow[b]{2}{*}{ Espèces } & \multirow[b]{2}{*}{ Familles } & \multicolumn{3}{|c|}{ Formations végétales } \\
\hline & & Cocoteraie & \begin{tabular}{|l} 
Fourré \\
littoral
\end{tabular} & Jachère \\
\hline Saccharum officinarum $\mathrm{L}$. & Poaceae & $\mathrm{x}$ & & \\
\hline Salacia baumannii Loes. & Celastraceae & $x$ & & \\
\hline Sansevieria liberica Gérôme \& Labroy & Agavaceae & & $x$ & \\
\hline Sauvagesia erecta L. & Ochnaceae & $x$ & & \\
\hline Scoparia dulcis L. & Scrophulariaceae & $x$ & & $x$ \\
\hline Sesamum radiatum Schumacher & Pedaliaceae & $x$ & & \\
\hline Sida cordifolia L. & Malvaceae & $x$ & $x$ & $x$ \\
\hline Sida linifolia Juss. ex Cav. & Malvaceae & $x$ & & \\
\hline Solanum lycopersicum L. & Solanaceae & $x$ & $x$ & \\
\hline Spermacoce ruelliae DC. & Rubiaceae & $x$ & & \\
\hline Spermacoce stachydea DC. & Rubiaceae & $x$ & & \\
\hline Spigelia anthelmia L. & Loganiaceae & & & $x$ \\
\hline Sporobolus pyramidalis P.Beauv & Poaceae & $x$ & $x$ & $x$ \\
\hline Stachytarpheta cayennensis (L.C. Rich.) Vahl. & Verbenaceae & $x$ & & $x$ \\
\hline Sterculia tragacantha Lindl. & Sterculiaceae & $x$ & & $x$ \\
\hline Stipularia africana P. Beauv. & Rubiaceae & $x$ & & $x$ \\
\hline Strophanthus gratus (Wall. \& Hook.) Baill. & Apocynaceae & $\mathrm{x}$ & $x$ & \\
\hline Strophanthus hispidus DC. & Apocynaceae & $x$ & $x$ & \\
\hline Stylosanthes fruticosa (Retz.) Alston & Fabaceae & $x$ & & $x$ \\
\hline Tapinanthus banguensis (Engl. and K. Krause) & Loranthaceae & $x$ & & \\
\hline Tephrosia villosa (L.) Pers. & Fabaceae & $x$ & $x$ & \\
\hline Terminalia catappa L. & Combretaceae & & $x$ & \\
\hline Tetracera alnifolia Willd. & Dilleniaceae & $x$ & & \\
\hline Tiliacora dinklagei Engl. & Menispermaceae & $x$ & & \\
\hline Triclisia patens Oliv. & Menispermaceae & $x$ & & \\
\hline Tridax procumbens $\mathrm{L}$. & Asteraceae & $x$ & $x$ & $x$ \\
\hline Triumfetta rhomboidea Jacq. & Tiliaceae & $\mathrm{x}$ & & $\mathrm{x}$ \\
\hline Vernonia cinerea (L.) Less. & Asteraceae & $\mathrm{x}$ & & $x$ \\
\hline Vitex grandifolia Gürke & Verbenaceae & $x$ & $x$ & \\
\hline Waltheria indica L. & Sterculiaceae & $x$ & & $x$ \\
\hline Ziziphus mauritiana Lam. & Rhamnaceae & & & $x$ \\
\hline Zornia latifolia Sm. var. Latifolia Sm & Fabaceae & $x$ & & $x$ \\
\hline
\end{tabular}

